\title{
Patient Engagement Drives the Positive Impact of Prehabilitation
}

Owen H. Brown, BS'; Brooke C. Kenney, $\mathrm{MPH}^{2,3}$; Brian A. Derstine, MS2, Dane R. Grenda, DO ${ }^{2,3,4}$; June A. Sullivan, MBA ${ }^{2,3,4}$; William C. Palazzolo, PA-C ${ }^{2,4}$; Stewart C. Wang, MD, PhD ${ }^{2,3,4}$; Michael J. Englesbe, MD ${ }^{2,3,4}$

Author Affiliations: 'University of Michigan Medical School, Ann Arbor, Ml; ${ }^{2}$ Michigan Surgical \& Health Optimization Program, University of Michigan, Ann Arbor, Ml; ${ }^{3}$ Morphomics Analysis Group, University of Michigan, Ann Arbor, Ml; ${ }^{4}$ Department of Surgery, University of Michigan, Ann Arbor, Ml

Author E-mail List: owenb

@med.umich.edu; nannab

@med.umich.edu; bderstin

@umich.edu; dgrenda@umich

.edu; jusulli@umich.edu; wpalazzo

@umich.edu; stewart@umich

.edu; englesbe@med.umich.edu

Corresponding Author:

Michael Englesbe, MD,

englesbe@med.umich.edu

Conflicts of interest:

Stewart C. Wang is an equity

stakeholder in Prenovo, LLC.

\section{ABSTRACT}

\section{Background}

The Michigan Surgical \& Health Optimization Program (MSHOP) is a multimodal surgical prehabilitation program. Enrollment in MSHOP has been associated with significant reductions in length of stay (LOS) and cost of care. It remains unclear whether a relationship exists between patient participation level in program interventions and the cost-saving impact.

\section{Methods}

We completed a retrospective analysis of patients enrolled in MSHOP between September 1, 2014, and December 31, 2015. Patients were categorized into two groups according to their degree of participation in the program: engaged or not engaged. We defined engaged patients as those who recorded steps in our system a minimum of three times per week for the majority ( $\geq 50 \%$ ) of weeks they were enrolled in the program. Univariate analysis was used to compare hospital costs and expected payer payment for surgical admissions within each participation group. Five program intervention variables were regressed against actual cost using a linear mixed model to determine the intervention-specific effect on engaged patients.

\section{Results}

A total of $82.7 \%$ of patients ( $N=$ 370) were engaged in MSHOP. For engaged patients, the median actual cost (IQR) was $\$ 18,448$ (range, $\$ 12,128-\$ 26,955)$, and the median expected payment (IQR) was $\$ 22,397$ (range, \$14,736-\$31,104), $P<0.0001$. No significant differences were observed in patients who were not engaged in the program. In multivariate analysis, total days enrolled and average weekly step count were associated with significant reductions in total cost among engaged patients $(P=0.0029$ and $P=0.0296$, respectively).

\section{Conclusions}

Patients who take an active role in surgical prehabilitation amplify the cost-savings impact compared to patients who merely enroll and participate. 


\section{Introduction}

$\mathbf{P}$ oor fitness capacity in surgical patients increases the risk of postoperative complications, can prolong recovery, and can significantly impact cost of care. ${ }^{1-4}$ Preparing patients for major surgery through targeted physical training, also known as prehabilitation, has garnered recent attention in the literature and may mitigate surgical costs. ${ }^{5}$ The Michigan Surgical \& Health Optimization Program (MSHOP) was founded with the intent to create a patient-centered approach to preoperative optimization that is made available to patients from the time of surgical decisionmaking until the operative procedure. ${ }^{6} \mathrm{MSHOP}$ combines physical, nutritional, and psychological guidance using a novel, home-based technology platform designed to encourage participation and compliance. Upon enrollment, patients are given a pedometer, an incentive spirometer, a DVD and brochure, and specific training from MSHOP personnel. More specific details about the program can be found at: http://www.med.umich.edu/ surgery/mshop/about.html.

Previous research has demonstrated that enrollment in MSHOP is associated with significant reductions in hospital length of stay (LOS) and cost of surgical care, ${ }^{5}$ but it remains unclear whether a relationship exists between patient participation level in certain MSHOP components and the cost-saving impact. Our study attempts to establish a correlation between these outcomes and the degree of patient participation beyond enrollment. Additionally, singular prehabilitation modalities have been independently associated with decreased total cost of surgical care..$^{7-12}$ However, in the context of a multimodal approach such as MSHOP, we must strategically parse the program components to determine the primary etiology of the cost-savings.

In this study, we analyze two key components of the MSHOP program that target fitness capacity of patients: logging daily step counts as tracked via a pedometer and recording use of an incentive spirometer. The first objective of this study was to determine which program components are positively associated with the cost-saving impact of MSHOP. The second objective was to determine the correlation between the level of participation in these program interventions and the cost of surgical admission for patients enrolled in MSHOP. We hypothesize that greater patient engagement in MSHOP will be associated with decreased surgical admission cost compared to expected insurance payments and that both intervention modalities contribute to this association.

\section{Methods Study Population}

We performed a retrospective analysis of patients enrolled in MSHOP between September 1, 2014, and December 31, 2015. Referring surgeons were encouraged to identify abdominal and thoracic surgery patients who they thought would benefit from the program. After assessing the distribution of surgical procedures and their actual costs, all nephrectomy and kidney-transplant patients were removed from the cohort. Index costs were determined to be unstable based on linkage to the transplant recipient as well as transplant drug treatment protocols. These protocols were deemed unrelated to prehabilitation and unnecessarily skewed the results. Inclusion was limited to patients having one of the following operations: colectomy, pneumonectomy, paraesophageal hernia repair, ventral hernia repair, esophagectomy, pancreatectomy, mastectomy, hepatectomy, cholecystectomy, esophagomyotomy, small bowel resection, gastrectomy, thoracotomy, video-assisted thoracoscopic surgery (VATS), femoral inguinal hernia, proctectomy, diverticulectomy, retroperitoneal resection, thymectomy, excision, hepaticojejunostomy, thyroidectomy, abdominal aortic aneurysm (AAA) repair, cystectomy, gastrojejunostomy, hysterectomy, lysis of adhesions, uterine myomectomy, ostectomy, chest wall reconstruction, and splenectomy. Patients who were unable to ambulate independently were excluded from this study due to their inability to complete the walking component of the program. Additionally, patients were excluded if they were 
suspended from the program (reasons included personal choice to leave, surgery postponed or canceled, and patient status changed) or if complete cost data was not available.

Patients were categorized into two groups according to their degree of participation in the program: engaged or not engaged. We defined engaged patients as those who recorded steps into our system a minimum of three times per week for the majority ( $\geq 50 \%$ ) of weeks they were enrolled in the program. Only complete weeks (seven consecutive days) were considered when determining patient engagement status. For example, if the enrollment period for a patient was four weeks and four days, engagement was only evaluated for the first four weeks. Also, when we assessed engagement, we did not consider compliance with the spirometry component of the program because this component is used only in the final two weeks of patient enrollment.

This study was approved by the University of Michigan Institutional Review Board; patient consent was waived.

\section{Outcome Measurements and Analysis}

We used inpatient and outpatient financial data associated with the index surgery stay to obtain our primary outcomes, actual cost, and expected payments. Professional fees were not included. These financial data were generated through Michigan Medicine's internal cost-accounting system, Transitions Systems Incorporated (TSI), which tracks resource utilization and assigns cost estimates to each resource. The system was used to produce actual cost (AC) as the sum of direct (patient care) and indirect (overhead) hospital costs and expected payments (EP) as the modeled revenue for reimbursement..$^{13}$ This method of cost accounting is generally accepted as the most accurate means of estimating the true costs of a healthcare encounter. ${ }^{14}$ The hospital generates a positive margin when the EP are greater than the AC.

All costs were inflation-adjusted to January 2015 dollars using the Bureau of Labor Statistics consumer price index. Because cost data distributions were skewed, we used the Wilcoxon signedrank test to compare the difference between $A C$ and EP within each participation category.

To understand the impact of MSHOP's key intervention components on index surgical cost, we limited the next part of our analysis to engaged patients. Meeting the minimum participation criteria allowed for a more homogenous sample to adequately measure the exposures. Walking and incentive spirometry activity in this cohort were assessed through the following: total number of days enrolled (day after registration until day before surgery), average weekly step count, rate of change in step count throughout the enrollment period, average number of breaths taken per spirometry entry, and how often the spirometer was utilized in the 14 days prior to surgery (\%). The rate of change was calculated using the patient's step count entries over the total entries made during enrollment. We defined spirometer utilization as the percentage of days the patient used their spirometer during the 14 days leading up to surgery. If the patient was enrolled less than a total of two weeks, we considered their enrollment period (in days) as the denominator to calculate this percentage. The previously listed surgical procedures were grouped into 12 general procedure buckets based on clinical judgement. To assess the statistical significance of the key MSHOP intervention components on actual cost, we used a generalized, linear mixed model with a random intercept. This was done because we anticipated that clustering would occur at the procedure level and each procedure bucket would produce similar actual costs. ${ }^{15,16}$ Actual cost was log-transformed to reduce the positive skew and regressed against the 5 intervention predictors and adjusted for gender, age, BMI, operative time, and procedure type (whereby procedure type was modeled as a random intercept).

A significance level of $a=.05$ was used. All statistical analysis was performed using the statistical software SAS 9.4. 


\section{Results}

There were a total of 536 MSHOP enrollments between September 1, 2014, and December 31, 2015. Complete cost data was not available for 129 patients, who were therefore excluded. One patient expired in the hospital after the surgical procedure and was excluded because the actual cost was not representative of the typical surgical stay. Eighteen patients were suspended from the program. Another 18 patients who had nephrectomy and kidney-transplant procedures were also excluded. Demographic and procedural information on the final cohort ( $N=370$ ) can be found in Table 1.

TABLE 1: Characteristics of MSHOP Cohort $(N=370)$

\begin{tabular}{ll}
\hline Demographics & Mean or Percent (Range) \\
\hline Female & $53 \%$ \\
\hline Male & $47 \%$ \\
\hline Age (years) & $62.4(20.0-89.9)$ \\
\hline BMI & $30.0(15.6-58.7)$ \\
\hline Operative time (min) & $212.7(17.0-686.0)$ \\
\hline Procedure Type & \\
\hline Colectomy & $17.0 \%$ \\
\hline Pneumonectomy & $14.0 \%$ \\
\hline Paraesophageal hernia & $10.8 \%$ \\
\hline Pancreatectomy & $9.5 \%$ \\
\hline Ventral hernia & $8.6 \%$ \\
\hline Esophagectomy & $6.8 \%$ \\
\hline Hepatectomy & $4.6 \%$ \\
\hline Mastectomy & $4.1 \%$ \\
\hline Cholecystectomy & $3.2 \%$ \\
\hline Esophagomyotomy & $3.0 \%$ \\
\hline Small bowel resection & $2.2 \%$ \\
\hline Other & $16.2 \%$ \\
\hline
\end{tabular}

Abbreviation: MSHOP, Michigan Surgical Health \&

Optimization Program

${ }^{1}$ Indicates procedures performed in $\leq 1.0 \%$ of the cohort

Based on our categorization, $82.7 \%$ of patients $(N=306)$ were engaged and $17.3 \%$ of patients ( $N=64$ ) were not engaged. For univariate analysis, engaged patients produced a greater reimbursement margin than not engaged patients and actual costs were found to be significantly lower than the expected payment. For engaged patients, the median actual cost (IQR) was $\$ 18,448$ (range, $\$ 12,128-\$ 26,955)$, and the median expected payment (IQR) was $\$ 22,397$ (range, $\$ 14,736-\$ 31,104$ ), 
$p<0.0001$ (Figure 1). For patients who were not engaged, median actual cost was \$22,793 (range, $\$ 13,531-\$ 32,712)$, and median expected payment was $\$ 23,305$ (range, $\$ 16,760-\$ 30,911$ ), with no significant difference between the two values.

Table 2 provides descriptive statistics of the intervention components among engaged patients. On average, engaged patients were enrolled in MSHOP for 37 days and recorded 25,655 steps per week (about 12.5 miles per week). The average step count decreased slightly during the program by about 20 steps per entry. Engaged patients used their spirometer $71.3 \%$ of their days enrolled, with an average 9 breaths per entry. Total days enrolled $(P=0.003)$ and average weekly step count $(P=$ 0.029) were significant predictors of actual cost in the multivariate analysis (Table 3). There was no significant relationship between actual cost and overall rate of change in step count, average weekly breath count, and percentage of spirometer usage. Since actual cost was log transformed, the significant variables were exponentiated to give a meaningful interpretation. For every additional day enrolled in the program, the average actual cost decreased by $0.24 \%$ when all other covariates were held constant. For every additional 10,000 steps taken per week (approximately 0.75 miles per day), there was about a 3.3\% reduction in average actual cost when all other covariates were held constant.

\section{Discussion}

Our study demonstrates a significant relationship between patient engagement, which includes walking and tracking steps, and the cost-saving impact of MSHOP. Further, patients who were enrolled in the program for a greater duration and those who were able to achieve high average weekly step counts were found to have lower

\section{Figure 1: Median Actual Cost and Expected Payments across MSHOP Participation Categories}

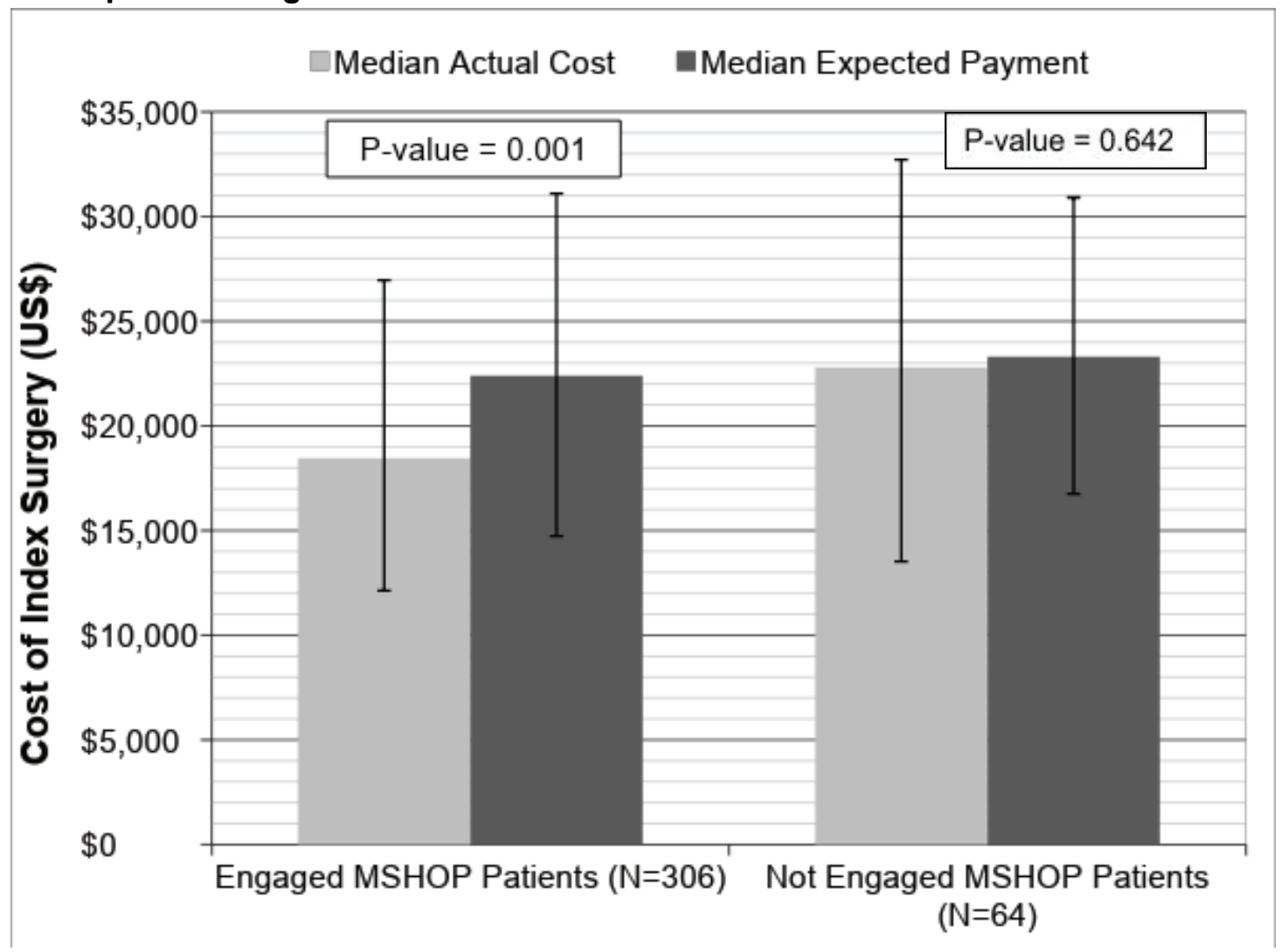

Numbers are unadjusted. Error bars represent interquartile range. 
TABLE 2: Descriptive Statistics of MSHOP Intervention Components among Engaged Patients $(\mathrm{N}=306)$

\begin{tabular}{lll}
$\begin{array}{l}\text { MSHOP Interven- } \\
\text { tion Component }\end{array}$ & Mean or Percent & Range \\
\hline Days Enrolled in MSHOP & 37 & $7-256$ \\
\hline Average Weekly Step Count & 25,655 & $421-126,826$ \\
\hline Rate of Change in Step Count & -20.27 & $-6122-2579$ \\
\hline Average Breath Count per Entry & 9 & $0-63$ \\
\hline Percentage of Spirometer Usage & $71.30 \%$ & $0.0 \%-100 \%$
\end{tabular}

Abbreviation: MSHOP, Michigan Surgical Health \& Optimization Program

${ }^{1}$ Rate of change represents the slope of each patient's step-count entries over the total entries made during enrollment.

TABLE 3: Model Results for MSHOP Intervention Components as Predictors of Actual Cost among Engaged MSHOP Patients $(\mathrm{N}=306)^{1}$

\begin{tabular}{|c|c|c|c|c|}
\hline Predictor & Estimate & $\begin{array}{l}\text { Lower Confidence } \\
\text { Interval }\end{array}$ & $\begin{array}{l}\text { Upper Confidence } \\
\text { Interval }\end{array}$ & P-value \\
\hline (Intercept) & 8358.2 & 4822.3 & $14,486.9$ & $<.0001$ \\
\hline Gender (male) & 1.027 & 0.912 & 1.160 & 0.648 \\
\hline BMI & 1.003 & 0.996 & 1.012 & 0.396 \\
\hline Age $^{*}$ & 1.005 & 1.000 & 1.010 & 0.046 \\
\hline Operative Time (per 10 minutes) ${ }^{*}$ & 1.029 & 1.024 & 1.035 & $<.0001$ \\
\hline Total Days Enrolled* & 0.998 & 0.997 & 0.999 & 0.003 \\
\hline Avg. Weekly Steps (per 10,000)* & 0.968 & 0.939 & 0.997 & 0.029 \\
\hline Rate of Change in Step Count ${ }^{2}$ & 0.999 & 0.999 & 1.000 & 0.703 \\
\hline Spirometer Usage (\%) & 0.871 & 0.724 & 1.047 & 0.142 \\
\hline Avg. Breath Count (per entry) & 1.001 & 0.996 & 1.007 & 0.597 \\
\hline
\end{tabular}

Abbreviation: MSHOP, Michigan Surgical Health and Optimization Program

${ }^{1}$ Estimates and confidence intervals have been exponentiated for easier interpretation. Results represent the multiplicative change in cost per unit increase in predictor.

${ }^{2}$ Rate of change represents the slope of each patient's step count-ntries over the total entries made during enrollment.

*Significant at the 0.05 alpha level. 
index surgical costs. These statistical relationships were not evident in nonengaged patients.

Improving the fitness of surgical candidates is achievable within a small preoperative window. Chen et al demonstrated improved functional walking capacity of elderly candidates with colorectal cancer surgery as measured by the 6-minute walk test (6MWT) following completion of a 4-week prehabilitation program. ${ }^{17}$ It is also clear that elevated fitness levels in surgical patients translates to reduced postoperative complications and better surgery-related outcomes. ${ }^{18}$ Together, these form the primary premise of prehabilitation: optimizing functional capacity should yield better outcomes, shorter recoveries, and lower health-care costs. Evaluation of our own program supported the basis of this theory, and comparable results have been demonstrated upon assessment of similar programs. ${ }^{5,19-21}$ To our knowledge, our study is the first to support the idea that, in addition to a formal program, patient-dependent factors play a significant role in the cost-savings impact of prehabilitation.

Data from this study has informed continuous MSHOP process improvement, identified opportunities to improve patient education, and provided costeffectiveness analysis to support implementation of MSHOP outside of our institution. Patients who take an active role and push themselves to improve their own fitness capacity are able to amplify the costsaving impact compared to patients who merely enroll and passively participate. With this information, providers should empower patients to take an active role in prehabilitation. Education efforts should focus particularly on promoting patient engagement and maximizing weekly step count throughout the program. Most importantly, hospitals should encourage patients to complete prehabilitation programs prior to elective surgery and invest in these programs based on the positive effects on margin and the low cost of program implementation. Likewise, payers should develop ways to enable provider billing for prehabilitation, which has the potential to reduce insurance costs and facilitate expansion of these yet uncommon programs.
Our study has several limitations. First, more patients participated in the program than were included in our analysis. Our data may not be representative of all MSHOP patients, thereby limiting the utility of this study in informing broad, programlevel analysis on cost effectiveness. Due to incomplete step-count tallies, we were forced to exclude many patients. Despite MSHOP implementation in a variety of specialties and care pathways, our analysis was performed in only a single academic institution. Our results do not suggest similar success will be achieved upon implementation in other centers when taking into account population or facility differences. As such, our further work, funded by the Health Care Innovation Award from the Center for Medicare and Medicaid Innovation, will focus on analysis to determine reproducibility in other institutions. The most important limitation is that, although we employed a prospective approach to assessment and data collection for patients receiving the MSHOP intervention, we have demonstrated only the association between engagement and costreduction. Many other clinical trials in prehabilitation used a more rigorous technique that allowed identification of causal relationships.22 Finally, patientreported outcomes may be a confounding variable influencing the degree of engagement and this was not rigorously collected or included in our analysis.

MSHOP enrollment referrals are currently left to surgeon discretion regarding whether a patient would or would not benefit from participation. In some patient populations, this decision most likely has basis in the safety of and risks related to prehabilitation. Symptoms relating to patient medical conditions as well as age-related frailty may pose significant limitations on achievable exercise intensity and thus nullify any potential benefit. These patients may still stand to benefit from prehabilitation; however, the appropriate patient-centered intervention modalities have yet to be developed for them. Additionally, further work evaluating the necessity of the incentive spirometry component may be considered, as this component was not associated with any significant cost savings. This 
will aid in resource optimization and minimizing program cost, as we continue toward the goal of MSHOP implementation on the state-wide level.

\section{Acknowledgments}

The authors acknowledge support from the National Institutes of Health $(\mathrm{NIH})$ grant 5T35HL007690-33; a Student Award Program from the Blue Cross Blue Shield of Michigan Foundation; and a Health Care Innovation Award from the Center for Medicare \& Medicaid Innovation.

\section{References}

1. Mastracci TM, Carli F, Finley RJ, et al. Effect of preoperative smoking cessation interventions on postoperative complications. J Am Coll Surg. 2011;212(6):1094-1096.

2. Dresler CM, Bailey $M$, Roper CR, et al. Smoking cessation and lung cancer resection. Chest. 1996;110(5):1199-1202.

3. Peng PD, Van Vledder MG, Tsai S, et al. Sarcopenia negatively impacts short-term outcomes in patients undergoing hepatic resection for colorectal liver metastasis. Hpb. 2011;13(7):439-446.

4. Lieffers JR, Bathe OF, Fassbender K, et al. Sarcopenia is associated with postoperative infection and delayed recovery from colorectal cancer resection surgery. Br J Cancer. 2012;107(6):931-936.

5. Englesbe M, Grenda D, Sullivan J, et al. The Michigan Surgical Home and Optimization Program is a scalable model to improve care and reduce costs. Surgery. 2017;161(6):1659-1666.

6. Michigan Surgical \& Health Optimization Program. http://www.med.umich.edu/surgery/mshop/.

7. Valkenet $K$, van de Port IG, Dronkers JJ, de Vries WR, Lindeman E, Backx FJ. The effects of preoperative exercise therapy on postoperative outcome: a systematic review. Clin Rehabil. 2011;25(2):99-111.

8. Pouwels S, Fiddelaers J, Teijink JAW, et al. Preoperative exercise therapy in lung surgery patients: A systematic review. Respir Med. 2015;109(12):1495-1504.

9. Carli F, Zavorsky GS. Optimizing functional exercise capacity in the elderly surgical population. Curr Opin Clin Nutr Metab Care. 2005;8(1):23-32.

10. Dronkers JJ, Lamberts $H$, Reutelingsperger IMMD, et al. Preoperative therapeutic programme for elderly patients scheduled for elective abdominal oncological surgery: a randomized controlled pilot study. Clin Rehabil. 2010;24(7):614-622.

11. Hoogeboom TJ, Dronkers JJ, van den Ende $\mathrm{CH}_{\text {, }}$ Oosting E, van Meeteren NL. Preoperative therapeutic exercise in frail elderly scheduled for total hip replacement: a randomized pilot trial. Clin Rehabil. 2010;24(10):901-910.

12. Hoogeboom TJ, Dronkers JJ, Hulzebos E, van Meeteren NL. Merits of exercise therapy before and after major surgery. Curr Opin Anaesthesiol. 2014;27(2):161-166.

13. Sheetz KH, Waits SA, Terjimanian MN, et al. Cost of major surgery in the sarcopenic patient. J Am Coll Surg. 2013;217(5):813-818.

14. Pronovost, P, Angus DC. Cost reduction and quality improvement: it takes two to tango. Crit Care Med. 2000;28(2):581-583.

15. Gerbershagen HJ, Pogatzki-Zahn E, Aduckathil S, et al. Procedure-specific risk factor analysis for the development of severe postoperative pain. Anesthesiology. 2014;120(5):1237-1245.

16. Bouwmeester W, Twisk JW, Kappen TH, et al. Prediction models for clustered data: comparison of a random intercept and standard regression model. BMC Med Res Methodol. 2013;13(1):19.

17. Chen BP, Awasthi R, Sweet SN, et al. Four-week prehabilitation program is sufficient to modify exercise behaviors and improve preoperative functional walking capacity in patients with colorectal cancer. Support Care Cancer. 2017;25(1):33-40.

18. Myers JN, Fonda $\mathrm{H}$. The impact of fitness on surgical outcomes: the case for prehabilitation. Curr Sports Med Rep. 2016;15(4):282-289.

19. Mayo NE, Feldman L, Scott S, et al. Impact of preoperative change in physical function on postoperative recovery: argument supporting prehabilitation for colorectal surgery. Surgery. 2011;150(3):505-514.

20. Santa Mina D, Clarke H, Ritvo P, et al. Effect of totalbody prehabilitation on postoperative outcomes: a systematic review and meta-analysis. Physiother (United Kingdom). 2014;100(3):196-207.

21. Moran J, Guinan E, McCormick P, et al. The ability of prehabilitation to influence postoperative outcome after intra-abdominal operation: a systematic review and meta-analysis. Surg (United States). 2016;160(5):1189-1201.

22. Carli F, Charlebois P, Stein B, et al. Randomized clinical trial of prehabilitation in colorectal surgery. $\mathrm{Br}$ J surg. 2010;97(8):1187-1197. 